\title{
COMPARISON OF STANDARD STATISTICAL THERMODYNAMICS WITH GENERALIZED STATISTICAL THERMODYNAMICS RESULTS FOR ISING CHAIN
}

\author{
U. Tirnakli, D. Demirhan and F. Büyükkiliç \\ Department of Physics, Faculty of Science, Ege University \\ 35100, Bornova İzmir, Turkey
}

(Received August 7, 1996; revised version December 18, 1996)

In this study, the internal energy and the specific heat of the one-dimensional Ising model obtained in the frame of the generalized statistical thermodynamics by Andrade, were somewhat extended and compared with the internal energy and the specific heat which were calculated in the standard (conventional) statistical thermodynamics. In the $q \rightarrow 1$ case, approaching of the generalized internal energy and the specific heat to the standard well-known results, were illustrated by numerical applications. In addition to these, $N \gg 1$ case was also investigated.

PACS numbers: 05.20.-y, 05.50.+q

\section{Introduction}

Boltzmann-Gibbs (BG) statistics is essentially derived from the standard Shannon entropy

$$
S_{1}=-k_{\mathrm{B}} \sum_{i=1}^{W} p_{i} \log p_{i}
$$

which is an extensive and concave quantity (the subindex 1 of $S$ will be defined afterwards). Although BG statistics is suitable for handling a large number of physical systems, it fails whenever

- the range of the microscopic interactions is large compared to the linear size of the macroscopic systems (long-range interactions);

- the time range of the microscopic memory is large compared to the observation time (non-Marcovian processes).

These kinds of violations are met for a long time in gravitation [1], magnetic systems [2], anomalous diffusion [3] and surface tension problems [4]. The way out from these problems seems to be nonextensive statistical thermodynamics which must be a generalization of the BG statistics in a manner which allows a correct 
description of the nonextensive physical systems as well. The study of nonextensive formalisms is very vivid nowadays in physics and these formalisms keep growing along two apparently different lines: generalized statistical thermodynamics (GST) and quantum group-like approaches (QGA). Although they seem to be very different from each other, recently it is pointed out that there exists a connection between them [5]. Here, the former one will be the subject of interest and the latter is completely out of the scope of the present study.

GST was proposed by Tsallis in 1988 [6]. The multifractal inspired entropy which was expressed by Tsallis in the form

$$
S_{q}=k \frac{1-\sum_{i=1}^{W} p_{i}^{q}}{q-1}
$$

is a generalized entropy having the properties of positivity, equiprobability, concavity and irreversibility. Furthermore, the standard additivity property has also been appropriately generalized (in the $q \neq 1$ case it becomes nonextensive). In Eq. (1), $k$ is a positive constant, $q$ (Tsallis $q$-index) is a real constant associated with the number of measurement in the multifractal theory and $W$ is the total configuration number. Commencing with the entropy definition of Eq. (1), a canonical distribution

$$
p_{i}=\frac{\left[1-\beta(q-1) \varepsilon_{i}\right]^{\frac{1}{q-1}}}{Z_{q}}, \quad Z_{q}=\sum_{i=1}^{W}\left[1-\beta(q-1) \varepsilon_{i}\right]^{\frac{1}{q-1}}
$$

could be obtained for a system in a heat bath, by maximizing the entropy under suitable constraints [6].

Soon after Tsallis' work, the generalization of the conventional concepts in various fields of physics have rapidly proceeded. Among the successfully tackled concepts in the frame of this generalization, generalized thermostatistics [7], Boltzmann $H$-theorem [8, 9], Ehrenfest theorem [10], the distribution function of the classical and quantum gases [11, 12], Langevin and Fokker-Planck equations [13], Bogolyubov inequality [14], fluctuation-dissipation theorem [15], von Neumann equation [16], classical equipartition principle [17], Callen identity [18], Ising chain $[19,20]$, paramagnetic systems [21], infinite-range spin-1/2 Ising ferromagnet [22], mean-field Ising model [23], the generalized transmissivity for spin-1/2 Ising ferromagnet [24], self-dual planar Ising ferromagnet [25] and Planck radiation law $[26,27]$ could be enumerated.

On the other hand, this generalization has also been successfully used to overcome the failure of BG statistics in some physical applications such as stellar polytropes [28], the specific heat of the non-ionized hydrogen atom [29], Levy-like anomalous diffusion [30], $d=2$ Euler turbulence [31], solar neutrino problem [32] and velocity distribution of galaxy clusters [33].

Up to now, a very large number of works, which have been performed in the frame of GST, are devoted to the investigation of the magnetic systems.

As an example of these, the distribution function of the paramagnetic systems might be noted. In this study, for the paramagnetic systems exhibiting multifractal structures, the connection between the Tsallis $q$-index and the fractal dimension and the range of the values of the $q$-index have been established [21]. 
In another work [23], using the generalized Bogolyubov inequality, the free energy and the magnetization of mean-field Ising model have been obtained within GST and it is noticed that the range of the $q$ values are consistent with the one obtained in Ref. [21]. Moreover, the expression obtained for the critical temperature is exactly the same as the one found in Ref. [18].

Another example for the investigation of the magnetic systems in the frame of GST is the Ising chain which is the subject of the present work. In his first study relevant to this subject, Andrade calculated [19] the internal energy and the specific heat of the Ising chain by making use of the distribution given in Eq. (2) which had been given before by Tsallis. Later on, in his second investigation [20] he reiterated his calculations using the distribution

$$
p_{i}=\frac{\left[1-\beta(1-q) \varepsilon_{i}\right]^{\frac{1}{1-q}}}{Z_{q}},
$$

where the partition function, $Z_{q}$, is

$$
Z_{q}=\sum_{i=1}^{W}\left[1-\beta(1-q) \varepsilon_{i}\right]^{\frac{1}{1-q}} .
$$

At first sight, this distribution seems to be very similar to Eq. (2), only with a small redefinition of $q$, however, this is not the case since this change originates from the fact that in the definition of the second constraint used for maximizing the Tsallis entropy, $q$-expectation values have been used.

IIere, our motivation is to improve Andrade's first work [19] somewhat by considering $r=3$ case and to strengthen Andrade's paper by comparing the obtained results with the standard $(q=1)$ ones. In the present study, the generalized internal energy and specific heat $(q \neq 1)$ have been compared with the standard results. In the $q \rightarrow 1$ case, the transformation of the results obtained by GST to the standard results, have been observed. Moreover, by taking into account the $N \gg 1$ case, the approaching of the internal energy expressions to a constant value and thus vanishing of the specific heat has also been illustrated. In this manner, the present study supports the investigations of Andrade as well as emphasizes the fact that GST is one of the most favourable tools for the examination of the multifractal structures. (It is obvious that the other study of Andrade [20] could also be tackled in the same manner.)

\section{One-dimensional Ising model}

In the model established by Ising for the first time with the objective of explaining ferromagnetism [34], a two-valued spin variable $s_{i}$ taking the values of +1 or -1 has been allocated for each lattice site. The model Hamiltonian in one dimension, in the absence of the magnetic field, could be written in the following form:

$$
\mathcal{H}=-J \sum_{i=1}^{N} s_{i} s_{i+1}
$$

where $J$ is the exchange parameter and $N$ is the number of total lattice sites. 
The analytical solution of the model with the transfer matrix method of standard statistical thermodynamics (SST) using the Hamiltonian of Eq. (5) leads to the following expressions for the internal energy and the specific heat respectively [35]:

and

$$
\frac{E}{J}=-N \tanh (\beta J)
$$

$$
\frac{C}{k_{\mathrm{B}}}=N \beta^{2} J^{2} \operatorname{sech}^{2}(\beta J)
$$

\section{Obtaining some of the thermodynamic quantities of the Ising chain using GST}

The most prominent difficulty met in the solution of systems investigated with GST is the determination of the partition function. This situation originates from the nonextensivity property which arises for the case $q \neq 1$. Andrade wrote the Hamiltonian of Eq. (5) with a parameter $\bar{E}$ which calibrates the ground state energy of the system for the Ising chain in the following form:

$$
\mathcal{H}=\bar{E}-J \sum_{i=1}^{N} s_{i} s_{i+1}
$$

and by taking $\bar{E}=-N J$ such that the values of the energy levels would become nonpositive from $-2 N J$ to 0 , he expressed the partition function of the system in a solvable form within the frame of GST by combinatorial method. As a consequence, he established the following expression [19]:

$$
E_{r}=-N J-J \frac{\sum_{m=0}^{r} \sum_{s=0}^{r-m}\left(\frac{\beta J}{r}\right)^{r-s} \frac{r !}{s ! m !(r-m-s) !} \sum_{u=0}^{m+1} \zeta_{m+1, u} N^{r+u-m-s}}{\sum_{m=0}^{r} \sum_{s=0}^{r-m}\left(\frac{\beta J}{r}\right)^{r-s} \frac{r !}{s ! m !(r-m-s) !} \sum_{u=0}^{m} \zeta_{m, u} N^{r+u-m-s}},
$$

where $r=1 /(q-1)$.

By writing down the definitions

$$
\begin{aligned}
& \zeta_{m u}=\sum_{n=0}^{u} C_{m}(n) \gamma_{m-n, u-n}(-1)^{n}, \\
& C_{m}(n)=\frac{m !}{n !(m-n) !}, \\
& \gamma_{m n}=\sum_{s=0}^{m} \eta_{n s}^{m} \quad(m, n, s \geq 0), \\
& \eta_{n s}^{m+1}=\eta_{n-1, s-1}^{m}+(s-m-1) \eta_{n, s-1}^{m}+s \eta_{n s}^{m},
\end{aligned}
$$

the internal energy values for $r=1(q=2), r=2(q=1.5), r=3(q=1.33)$ from Eq. (9) could be written down as

$$
\begin{aligned}
& E_{1}=-N J-\frac{\beta J^{2} N}{\beta J N+1}, \\
& E_{2}=-N J-\frac{\beta J^{2} N\left(\frac{1}{2} \beta J N+1\right)}{\frac{1}{4} \beta J N[\beta J(N+1)]+1},
\end{aligned}
$$




$$
E_{3}=-N J-\frac{\beta J^{2} N\left[\frac{\beta^{2} J^{2}}{9}\left(N^{2}+N-\frac{2}{3}\right)+\frac{2}{3} \beta J N+1\right]}{\frac{1}{3} \beta J N\left[\frac{\beta^{2} J^{2}}{3}\left(N+\frac{N^{2}}{3}\right)+\beta J(N+1)\right]+1} .
$$

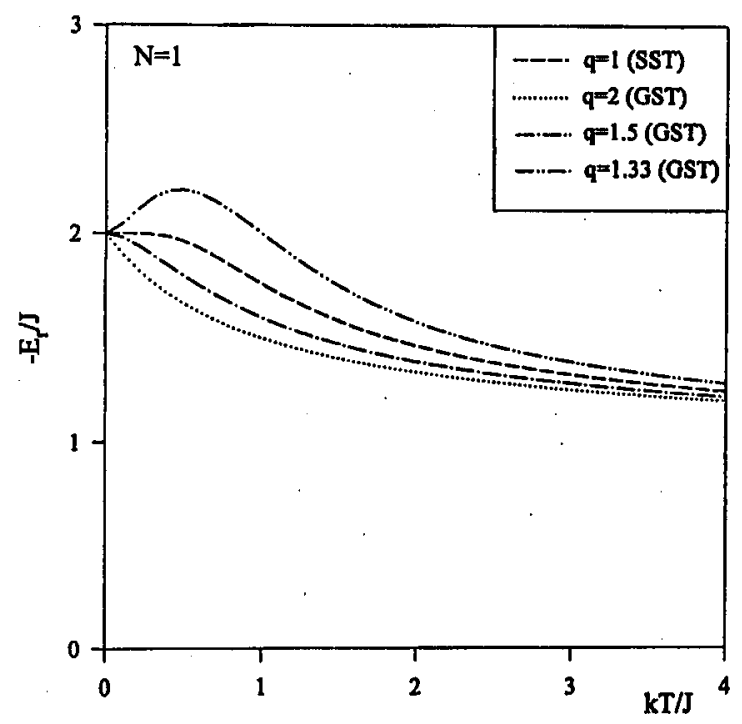

Fig. 1. The variation of the internal energy with the reduced temperature for $N=1$.

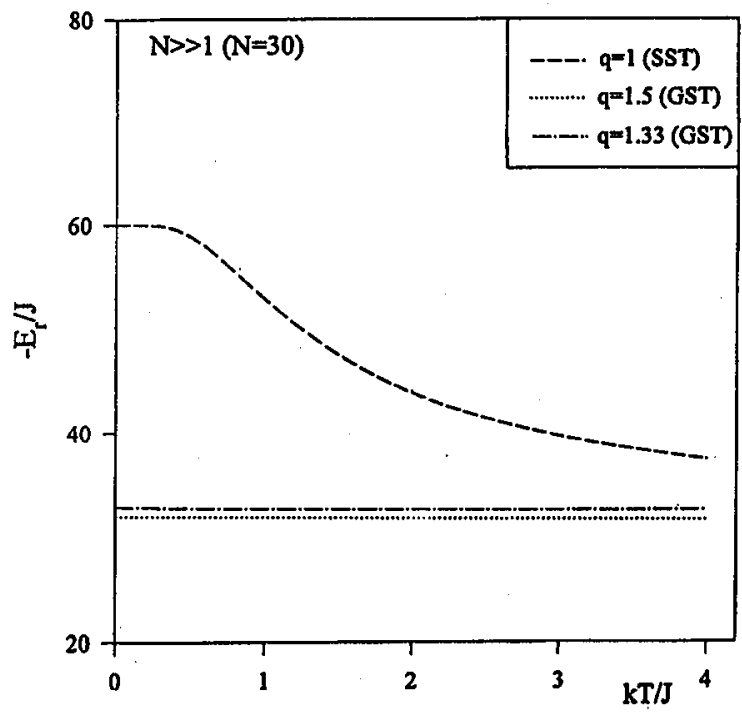

Fig. 2. The variation of the internal energy with the reduced temperature for $N \gg 1$, namely $N=30$. 
The variations of the internal energy with respect to the reduced temperature $1 / \beta J$ for Eqs. (14)-(16) of GST and Eq. (6) of SST were illustrated in Fig. 1. In accordance with the expectations, for increasing $r$ values ( $q$ values decreasing to 1 ) the behaviour of the internal energy curve approaches to that of the analytical solution of SST. Furthermore, in $N \gg 1$ case, as it is illustrated in Fig. 2, $E_{r}$ approaches to a constant value which is an unexpected result.

From the internal energy expressions given by Eqs. (14), (15) and (16), taking $r=1(q=2), r=2(q=1.5), r=3(q=1.33)$, the following conclusions could respectively be obtained for the specific heat:

$$
\begin{aligned}
& \frac{C_{1}}{k}=\frac{\beta J^{2}}{(1+\beta J N)^{2}}, \\
& \frac{C_{2}}{k}=\frac{4 \beta^{2} J^{2}\left[4+4 \beta J N+\beta^{2} J^{2} N(N-1)\right]}{\left[4+4 \beta J N+\beta^{2} J^{2} N(N+1)\right]^{2}}, \\
& \frac{C_{3}}{k}=9 \beta^{2} J^{2} \\
& \times \frac{\left[\beta^{4} J^{4}\left(N^{4}+N^{2}-2 N\right)-6 \beta^{3} J^{3}\left(N^{3}+3 N^{2}\right)-18 \beta^{2} J^{2}+108 \beta J N+81\right]}{\left[\beta^{3} J^{3}\left(N^{3}+3 N^{2}\right)+9 \beta^{2} J^{2}\left(N^{2}+N\right)+27\right]} .
\end{aligned}
$$

The variation of the specific heat with respect to the reduced temperature for $N=1$ was illustrated in Fig. 3. It was noticed that the curves plotted using Eqs. (17), (18) and (19) of GST for $r=1,2$ and 3 approach to the curve given by Eq. (7) for increasing $r$ values (while $q$ values decrease to 1 ), which was established

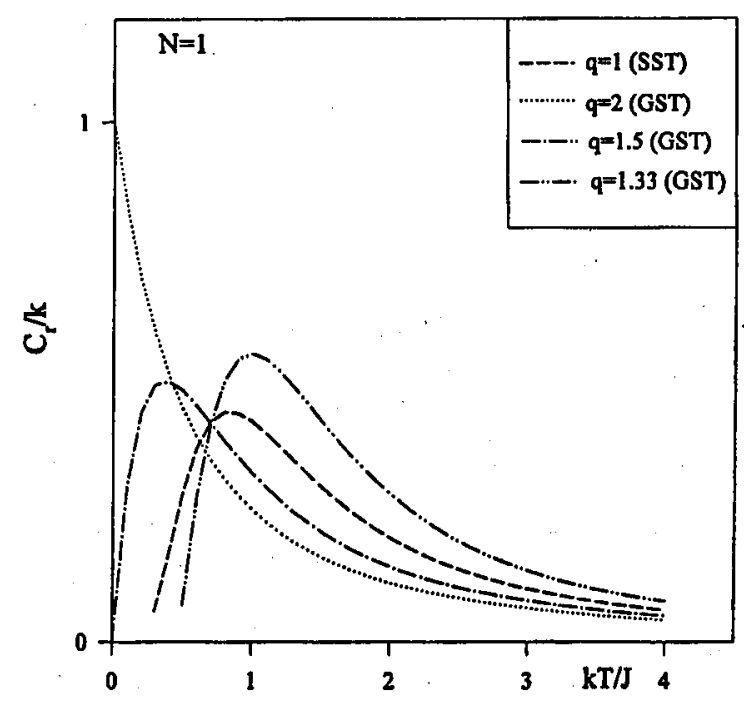

Fig. 3. The variation of the specific heat with the reduced temperature for $N=1$. 
by the transfer matrix method of SST. Furthermore, in $N \gg 1$ case, approaching of $E_{r}$ to a constant value naturally causes $C_{r}$ to vanish.

\section{Conclusions}

In this study, the expressions which were obtained in GST for the internal energy and the specific heat of the Ising chain were compared with the corresponding relations in SST. $q$ could be regarded as a parameter belonging to the internal encrgy and the specific heat. It was clearly shown by numerical calculations that the internal energy and the specific heat established for the Ising chain in the frame of GST approach to the conventional results obtained by the Boltzmann-Gibbs distribution in the $q \rightarrow 1$ (increasing $r$ ) case. These conclusions conform to our expectations such as the transformation of the Tsallis entropy (Eq. (1)) to the Shannon entropy. The parametric effect of $q$ was brought to a concrete basis with the aid of the plots. The verification of the internal energy and the specific heat expressions brought in by Andrade were supported by the standard results obtained by the transfer matrix method. In addition to these, the $N \gg 1$ case was also considered; the explicit approaching of the internal energy to a constant value which in turn leads to the vanishing of the specific heat was graphically illustrated.

\section{Acknowledgment}

The authors would like to thank Prof. C. Tsallis for his helpful comments.

\section{References}

[1] A.M. Salzberg, J. Math. Phys. 6, 158 (1965); W.C. Saslaw, Gravitational Physics of Stellar and Galactic Systems, Cambridge University Press, Cambridge 1985, p. 217; P.T. Landsberg, J. Stat. Phys. 35, 159 (1984); D. Pavon, Gen. Relativ. Gravit. 19, 375 (1987).

[2] B.J. Hiley, G.S. Joyce, Proc. Phys. Soc. 85, 493 (1965).

[3] M.F. Shlesinger, B.D. Hughes, Physica A 109, 597 (1981); E.W. Montroll, M.F. Shlesinger, J. Stat. Phys. 32, 209 (1983).

[4] J.O. Indekeu, Physica A 183, 439 (1992); J.O. Indekeu, A. Robledo, Phys. Rev. E 47, 4607 (1993).

[5] C. Tsallis, Phys. Lett. A 195, 329 (1994).

[6] C. Tsallis, J. Stat. Phys. 52, 479 (1988).

[7] E.M.F. Curado, C. Tsallis, J. Phys, A 24, L69 (1991); Erratum 24, 3187 (1991); 25, 1019 (1992).

[8] A.M. Mariz, Phys. Lett. A 165, 409 (1992).

[9] J.D. Ramshaw, Phys. Lett. A 175, 169, 171 (1993).

[10] A. Plastino, A.R. Plastino, Phys. Lett. A 177, 177 (1993).

[11] F. Büyükkılıç, D. Demirhan, Phys. Lett. A 181, 24 (1993).

[12] F. Büyükkılıc, D. Demirhan, A. Gülec, Phys. Lett. A 197, 209 (1995).

[13] D.A. Stariolo, Phys. Lett. A 185, 262 (1994).

[14] A. Plastino, C. Tsallis, J. Phys. A 26, L893 (1993).

[15] E.P. da Silva, C. Tsallis, E.M.F. Curado, Physica A 199, 137 (1993); 203, E160 (1994); A. Chame, E.M.L. de Mello, J. Phys. A 27, 3663 (1994). 
[16] A.R. Plastino, A. Plastino, Physica A 202, 438 (1994).

[17] A.R. Plastino, A. Plastino, C. Tsallis, J. Phys. A 27, 5707 (1994).

[18] E.F. Sarmento, Physica A 218, 482 (1995).

[19] R.F.S. Andrade, Physica A 175, 285 (1991).

[20] R.F.S. Andrade, Physica $A$ 203, 486 (1994).

[21] F. Büyükkılıç, D. Demirhan, Z. Phys. B 99, 137 (1995).

[22] F.D. Nobre, C. Tsallis, Physica A 213, 337 (1995).

[23] F. Büyükklıç, D. Demirhan, U. Tırnaklı, Physica $A$, in press.

[24] L.R. da Silva, H.E. Stanley, Physica A 234, 497 (1996).

[25] S.A. Cannas, C. Tsallis, Z. Phys. B 100, 623 (1996).

[26] C. Tsallis, F.C. Sa Barreto, E.D. Loh, Phys. Rev. E 52, 1447 (1995).

[27] U. Tirnaklı, F. Büyükkılı̧, D. Demirhan, Physica A, in press.

[28] A.R. Plastino, A. Plastino, Phys. Lelt. A 174, 384 (1993).

[29] L.S. Lucena, L.R. da Silva, C. Tsallis, Phys. Rev. E 51, 6247 (1995).

[30] P.A. Alemany, D.H. Zanette, Phys. Rev. E 49, R956 (1994); C. Tsallis, S.V.F. Levy, A.M.C. Souza, R. Maynard, Phys. Rev. Lett. 75, 3589 (1995); C. Tsallis, A.M.C. Souza, R. Maynard, in: Levy Flights and Related Phenomena in Physics, Eds. M.F. Shlesinger, U. Frisch, G.M. Zaslavsky, Springer, Berlin 1995, p. 269.

[31] B.M. Boghosian, Phys. Rev. E 53, 4754 (1996).

[32] G. Kaniadakis, A. Lavagno, P. Quarati, Phys. Lelt. B 369, 308 (1996).

[33] A. Lavagno, G. Kaniadakis, M. Rego-Monteiro, P. Quarati, C. Tsallis, Non-extensive Thermostatistical Approach of the Peculiar Velocity Function of Galaxy Clusters, 1996, preprint.

[34] E. Ising, Z. Phys. 31, 253 (1925).

[35] B.M. Mc Coy, T.T. Wu, The Two-dimensional Ising Model, Harvard University Press, Cambridge 1973. 